\title{
The Electromagnetic Unification of Gravitational Force by Chinese
}

\author{
Zi-Jian Cai \\ CaiFortune Consulting, No. 129, Building 6, Room 404, North Dongwu Road, Suzhou, China \\ Email:hrsh8@126.com
}

How to cite this paper: Cai, Z.-J. (2020) The Electromagnetic Unification of Gravitational Force by Chinese. Open Access Library Journal, 7: e6175.

https://doi.org/10.4236/oalib.1106175

Received: February 17, 2020

Accepted: March 6, 2020

Published: March 9, 2020

Copyright $\odot 2020$ by author(s) and Open Access Library Inc.

This work is licensed under the Creative Commons Attribution International License (CC BY 4.0).

http://creativecommons.org/licenses/by/4.0/

(c) (i) Open Access

\begin{abstract}
In the recent book "Electromagnetic Unification of Four Forces" (ISBN978-3659-76798-2), it was compiled the relevant progressions made by the world people in television while led notably by me and the Members in satellite networks from China, India and USA. It was the author Zi-Jian Cai and European people in television who proposed the gravitational mass of particle as the spatially localized electromagnetic structure, different from the Yang-Mills equation to unify the charge with field. Later, it was a Chinese with heavy Suzhou tone operating satellite who proposed the asymmetrical electromagnetic orienting force as attraction longer than repulsion accumulatively irrelevant to charge. Accordingly, it explained the gravitational field of Einstein's general relativity as the curve and red shift of electromagnetic wave by such force, with the changes in Einstein's physical time and space replaced by those in electromagnetic frequency and wavelength. For paradoxes/problems besides these main themes, it interpreted the constant gravitational $\mathrm{G}$ as resulting from identical mass frequency for the same quark. Because it was the constance of electromagnetic environment adopted by Chinese people to explain the irrelevance of light speed to movement in vacuum, plus the speed of neutrino of mass at least equal to that of light, it explained the special relativity by the changes in electromagnetic frequency and wavelength as in electromagnetic general relativity, with the Einstein's physical time and space replaced by the non-relative ideal time and space without electromagnetic interactions, while the interactions among electromagnetic particles and fields generating the Lorentz transformation.
\end{abstract}

\section{Subject Areas}

Theoretical Physics, Modern Physics, Special Theory of Relativity

\section{Keywords}

Electromagnetic Localized Mass Structure, Electromagnetic Orienting Force, 
Gravitational Field, Light Curve, Red Shift, Relativity, Ideal Time and Space, Speed of Neutrino

\section{Introduction}

For one form of energy present as the frequency of electromagnetic wave or frequency of light, such three other forces of energy as the strong force, weak force and gravitational force, should be unified with the electromagnetic force of light on energy. Recently, the author published the book "Electromagnetic Unification of Four Forces" (ISBN978-3-659-76798-2) [1], compiling the progressions of the great unification in physics. The author Zi-Jian Cai along with the Chinese Members, India Members, and US Members in the world satellite networks organized the movement and accomplished altogether with the anonymous people in worldwide television [1].

The most notable contributions made by Chinese in this movement fell into the unification of gravitational force with electromagnetic force. In this article, it is reviewed the relevant achievements and intended to make it convenient for reading and citing.

\section{The New Spatially Localized Electromagnetic Mass Structure}

Based on the fact that the $\gamma$ photon of high energy could transform with the gravitational mass in reciprocation [2] [3], the author Zi-Jian Cai and European people in television proposed that the mass of particle should possess the spatially localized electromagnetic structure. The mass electrical field and magnetic field of particle moved continuously and transformed reciprocally, but spatially this electromagnetic mass structure did not resemble that of photon which propagated away. Instead, it returned to the vicinity around its original point of departure via its electromagnetic movement and transformation [2] [3].

Different from the early Yang-Mills equation to unify the charge of particle with field [4], the spatially localized electromagnetic wave herein was adopted to form the mass structure for unifying the astronomic gravitational force with electromagnetic force.

After $\mathrm{Zi}$-Jian Cai led the sate-commune group to make this proposition in early October 2005, the activities in discussion and exchange of physics elicited the attention, comment and participation of people in the whole world with television as the common media.

The increase in mass during quark condensation [5] [6] strongly supports the spatially localized electromagnetic mass structure, because the mass increment can be easily explained by the electromagnetic conformational change [2] [3].

\section{The Electromagnetic Orienting Force}

On October 11, 2005, a member of Sate-Commune Group with heavy Suzhou 
tone simply calling himself as Zhejiang person (many residing in Suzhou) proposed the electromagnetic orienting force [2] [7]. When several electromagnetic particles or waves were close together, among their electrical fields there would occur the electromagnetic interactions with the same charge repelling and opposite charge attracting, or the electromagnetic fields of same direction attracting and opposite direction repelling. The attraction made the electromagnetic fields decrease in distance, increase in interactive strength and increase in interactive time. The repulsion made the electromagnetic fields increase in distance, decrease in interactive strength and decrease in interactive time. Such asymmetrical interactions made them generate a kind of net attractive force among the electromagnetic particles or waves, called as the electromagnetic orienting force [2] [7].

Because the electromagnetic orienting force results from the asymmetrical electromagnetic interactions as attraction longer than repulsion, it is a force from the accumulative effects of both attraction and repulsion. In this regard, accumulatively the electromagnetic orienting force is irrelevant to the characters of charges of particles, so that it is a good candidate of gravitational force.

\section{The Electromagnetic Gravitational Field}

It was suggested in the book that the electromagnetic mass waving entity of gravitational donor interacted in electromagnetic orientation with the electromagnetic waves mediating the gravity, making them turn in curve [2] [8]. While such orienting interaction fading away with the electromagnetic waves partially returning to the original status, the electromagnetic waves consumed energy and shifted red [2] [8]. Both of them manifested in sum as the phenomena of gravitational field [2] [8]. The photon and electromagnetic wave curving and shifting red in gravitational field directly supported this explanation [2] [8]. Besides, the increment of mass during condensation of quarks [5] [6] could only be explained by the electromagnetic conformational change of gravitational entity, with higher mass frequency affected more in coordination in gravitational attraction, supporting this mechanism of gravitational force.

Because this explanation of gravitational field with the electromagnetic interactions relies heavily on the curving and shifting red of electromagnetic wave by electromagnetic orientation, it is obviously the electromagnetic manifestations of Einstein's general relativity, but with the gravitational changes in relative physical time and space(as the frequency and length of respective physical clock and wavelength for measurement) replaced directly by those in electromagnetic frequency and wavelength.

To account for different elements have the same gravitational constant G, the author Cai explained that the gravitational force mainly resulted from the gravitational attraction by heavy quarks in atomic nuclei rather than light electrons among various elements, which were identical in mass frequency for the same quark [9]. 


\section{The Physical and Ideal Time and Space}

\subsection{The Mistakes of Einstein's Special Relativity}

On September 25, 2008, the Yangzhou People in China on television pointed out that the cosmic vacuum was just alike to other media in determining the light speed with their own electromagnetic characteristics. Even though the illuminant could move, the light speed determined by the constant electromagnetic media of vacuum was likewise fixed and unchangeable [10]. In this way different from Einstein, it was explained the phenomenon of special relativity that the light speed was irrelevant to the speed of illuminant. Also, the movement of illuminant should as well bring kinetic energy to the photon, which must be compensated or absorbed by the photon itself through its red shift or blue shift in wavelength [10].

Besides, it was demonstrated in updated experiments that the speed of neutrino was at least equal to that of light [11]. In consideration of that the neutrino possessed the mass, such result was unfavorable to the rationality of Einstein's special relativity.

Because of the mistakes in physics for Einstein's special relativity [10] [11], it is certainly necessary to revise the special relativity of Einstein to explain the assayed effects of Lorentz change on time and length from movement.

\subsection{The Non-Relativity of Ideal Time and Space}

Because the electromagnetic general relativity results from the electromagnetic interactions, it is necessary to inspect the ideal time and space in reference system without electromagnetic interactions.

In the book "Electromagnetic Unification of Four Forces" (ISBN978-3-65976798-2) [1], it was demonstrated the non-relativity of ideal time and space without electromagnetic interactions [2] [12]. The ideal reference system $O$ was stationary, the ideal reference system $\mathrm{A}$ and $\mathrm{B}$ moved at constant velocity $\mathrm{v}$ and $-\mathrm{v}$ respectively. From the reference system $\mathrm{O}$ to see $\mathrm{A}$, the relative velocity was $\mathrm{v}$. If the axial unit of time itself possessed relativity, its relative time changed $t$. From the reference system $\mathrm{O}$ to see $\mathrm{B}$, the relative velocity was - $\mathrm{v}$, and its relative time also changed $t$. In this regard, the two axial units of time of reference system $A$ and $B$ were equal and the same. Again from the reference system $B$ to see $A$, the relative velocity of reference system $A$ was $2 \mathrm{v}$, while the change in relative time was close to $2 t$, different from the situation observed from the reference system $O$. These two states were concurrent but contradictory. The only explanation was that the difference in value for these axial units of time $t=0$. With the same method, it could likely be deduced that the value of difference for the axial units of space $x=0$. To solve these contradictions and paradoxes, it was necessary to conclude that there was no relativity in time and space [2] [12].

\subsection{The Electromagnetic Special Relativity}

The time and space in Einstein's relativity is the relative physical time and space 
using electromagnetic units to measure by the frequency and length of respective physical clock and wavelength. In the electromagnetic gravitational field, it is not necessary to change the reference system, so that the changes in electromagnetic frequency and wavelength from electromagnetic orientation just correspond to the gravitational changes in relative physical time and space of general relativity.

In the special relativity of Einstein, the situation is different because the relative electromagnetic (physical) unit of time or space changes following movement of reference system at constant velocity. However, as mentioned above, Einstein made mistakes in explaining the irrelevancy of light speed to movement in vacuum [10] while the neutrino of mass was able to travel at speed at least equal to that of light [11]. Therefore, it is necessary to revise the special relativity of Einstein.

The ideal time and space without electromagnetic interactions manifest non-relativity [2] [12], different from the electromagnetic (physical) unit of time and space in Einstein's special relativity. On the other hand, the Lorentz effects on time and length from movement were the key phenomena of special relativity, necessary to explain. Obviously, the discrepancies result from the difference of ideal time and space without electromagnetic interactions and the electromagnetic (physical) unit of time and length in special relativity.

In the electromagnetic gravitational field, the changes in electromagnetic frequency and wavelength correspond to the gravitational changes in relative physical time and space of general relativity. Because the special relativity and general relativity adopt the same electromagnetic (physical) unit of time or space, the changes in relative physical time and space in general relativity, or the changes in electromagnetic frequency and wavelength in electromagnetic gravitational field, are the same to the changes in relative physical time and space in special relativity. In this regard, herein it is interpreted the special relativity of Lorentz transformation with the electromagnetic general relativity.

\subsection{The Electromagnetic Special Relativity without Paradox}

As mentioned above, by the presence of contradiction and paradox, it was proven that there was no relativity for the ideal time and space by themselves moving at constant velocity without electromagnetic interactions [2] [12]. Herein, it is necessary to demonstrate that, in the ideal reference system without relativity in time and space but plus electromagnetic interactions, there would be no longer presence of contradiction and paradox, so that the electromagnetic special relativity does not face the same difficulty.

The electromagnetic fields of atoms, earth and sun can approximately be simplified as the centric radial electrical field. To demonstrate this problem, the US operators of satellites likewise also adopted the above three ideal reference systems without electromagnetic interactions, among which $\mathrm{O}$ was stationary, $\mathrm{A}$ moved at constant velocity $\mathrm{v}$ and $\mathrm{B}$ at constant velocity $-\mathrm{v}$ [13]. Particle $\mathrm{X}$ moved at constant velocity $\mathrm{v}$ and particle $\mathrm{Y}$ at constant velocity $-\mathrm{v}$ [13]. At the 
center of ideal reference system $\mathrm{O}$ there was a centric radial electrical field $\mathrm{E}$ [13]. Particle X and Y both formed electromagnetic orientation with the electrical field E. As movement of particle X and Y made their electromagnetic orientation with $\mathrm{E}$ shift blue, compared to their own stationary state, their wavelength both decreased as a change of $\mathrm{x}$ in equal [13]. There was no relative difference in wavelength for particle $\mathrm{X}$ as compared to $\mathrm{Y}$. This was the situation observed in the ideal reference system $O$. To see from the reference system $B$, the moving velocity of particle $Y$ was 0 , while that of $X$ was $2 \mathrm{v}$. At this time, the centric radial electrical field $\mathrm{E}$ in the ideal reference system $\mathrm{O}$ acquired moving velocity, which was $\mathrm{v}$ [13]. Thereby, the velocity of particle $\mathrm{X}$ relative to the centric radial electrical field $\mathrm{E}$ was $\mathrm{v}$. The movement made its electromagnetic orientation with $\mathrm{E}$ shift blue, which decreased its wavelength by $\mathrm{x}$ [13]. The velocity of particle $\mathrm{Y}$ relative to the centric radial electrical field $\mathrm{E}$ was $-\mathrm{v}$. The movement made its electromagnetic orientation with $\mathrm{E}$ shift blue, which also decreased its wavelength by $\mathrm{x}$ [13]. Both particles changed in wavelength by the same $\mathrm{x}$, which was the same as that observed in reference system $\mathrm{O}$, and was not in contradiction with each other [13]. Accordingly, with electromagnetic interactions, it was successful in solving the contradiction and paradox in special relativity that observation in different reference systems might lead to different results.

\section{Discussions}

In this article, it is specially reviewed the electromagnetic unification of gravitational field in the recently published book "Electromagnetic Unification of Four Forces" (ISBN978-3-659-76798-2) [1], and is intended to make it easy to read and cite. The Chinese made the major contributions to these achievements.

Different from the Yang-Mills equation to unify the charge of particle with field [4], the author Zi-Jian Cai and European people in television proposed the spatially localized electromagnetic wave to form the mass structure for unifying the astronomic gravitational force with electromagnetic force [2] [3], based on the mutual transformation of $\gamma$ photon of high energy and the gravitational mass [2] [3]. The increase in mass during quark condensation [5] [6] can be explained by this electromagnetic mass structure via electromagnetic conformational change.

A member of Sate-Commune Group with heavy Suzhou tone proposed the electromagnetic orienting force [2] [7], with the electromagnetic fields of opposite charge or same direction attracting to decrease the distance, increase the interactive strength and time, while the electromagnetic fields of same charge or opposite direction repelling to increase the distance, decrease the interactive strength and time. Such asymmetrical interactions made them generate a kind of net attractive force as the electromagnetic orienting force [2] [7]. Because the electromagnetic orienting force is a force from the accumulative effects of both attraction and repulsion, it is irrelevant to the characters of charges of particles and becomes a good candidate of gravitational force. 
With the electromagnetic orienting force, the gravitational field of Einstein's general relativity was suggested to manifest as the curve and red shift of electromagnetic wave, while the changes in time and space of Einstein's general relativity were replaced by those in electromagnetic frequency and wavelength for electromagnetic gravitational field [2] [8]. The photon and electromagnetic wave curving and shifting red in gravitational field [2] [8] as well as the increment of mass during condensation of quarks [5] [6] can both support this explanation.

The mass as spatially localized electromagnetic structure, the electromagnetic orienting force, and the electromagnetic gravitational field of general relativity are the main themes of electromagnetic unification of gravitational force (Table 1), while there are still some related paradoxes and problems required to be solved (Table 1).

For the same constant of gravitational G among different elements, the author Cai explained that the gravitational force mainly resulted from the quarks rather than electrons, with the same mass frequency for the same quark [9].

The special relativity of Einstein has encountered new difficulties. On the one hand, the Yangzhou People in China on television pointed out that, even though

Table 1. Clarification of contents for electromagnetic unification of gravitational force.

\begin{tabular}{|c|c|c|c|}
\hline Contents & Main theme & Paradoxes & Explanations \\
\hline $\begin{array}{l}\text { Mass as spatially localized } \\
\text { electromagnetic structure }\end{array}$ & Yes & & $\begin{array}{l}\text { 1) The reciprocal transformation of } \gamma \text { photon and } \\
\text { mass. } \\
\text { 2) The increase in mass in quark condensation. }\end{array}$ \\
\hline The electromagnetic orienting force & Yes & & $\begin{array}{l}\text { Asymmetrical as attraction longer than repulsion in } \\
\text { time. }\end{array}$ \\
\hline $\begin{array}{l}\text { The curve and red shift of } \\
\text { electromagnetic wave in electromagnetic } \\
\text { gravitational field }\end{array}$ & Yes & & $\begin{array}{l}\text { 1) The electromagnetic wave and photon curving } \\
\text { and shifting red in gravitational field of general } \\
\text { relativity. } \\
\text { 2) The electromagnetic conformational change to } \\
\text { explain increment of mass in quark condensation. }\end{array}$ \\
\hline $\begin{array}{l}\text { The physical time/space of general } \\
\text { relativity replaced by electromagnetic } \\
\text { frequency/wavelength }\end{array}$ & Yes & & $\begin{array}{l}\text { The electromagnetic wave and photon curving and } \\
\text { shifting red in gravitational field of general } \\
\text { relativity. }\end{array}$ \\
\hline Same $\mathrm{G}$ for various matters & & Yes & Same mass for same quark \\
\hline $\begin{array}{l}\text { Physical time/space of general relativity } \\
\text { same as those in special relativity }\end{array}$ & & Yes & As from Einstein's original theories. \\
\hline $\begin{array}{l}\text { Speed of light/neutrino violating special } \\
\text { relativity of Einstein }\end{array}$ & & Yes & $\begin{array}{l}\text { 1) Light speed affected by electromagnetic constants } \\
\text { to explain moving illuminant. } \\
\text { 2) Neutrino speed at least equal to or higher than } \\
\text { light. }\end{array}$ \\
\hline Nonrelativity of ideal time/space & & Yes & $\begin{array}{l}\text { Suitable to ideal time/space without physical } \\
\text { interactions }\end{array}$ \\
\hline $\begin{array}{l}\text { Special relativity of Lorentz time/space } \\
\text { transformation }\end{array}$ & & Yes & Resulting from electromagnetic interactions \\
\hline
\end{tabular}


the illuminant could move, the light speed determined by the constant electromagnetic media of vacuum was fixed and unchangeable [10], different from Einstein. On the other hand, updated experiments have shown that the neutrino possessing mass might travel at speed at least equal to that of light [11], directly challenging the correctness of Einstein's special relativity.

In the book "Electromagnetic Unification of Four Forces" (ISBN978-3-65976798-2) [1], it was demonstrated the non-relativity of ideal time and space without electromagnetic interactions [2] [12]. Such discrepancies should result from the difference of ideal time or space without electromagnetic interactions and the electromagnetic (physical) unit of time or length in special relativity. The US operators of satellites overcame the plausible non-relativity of electromagnetic moving particles by using their electromagnetic interactions with the centric radial electrical field [13].

Because of the electromagnetic (physical) unit of time and space in general relativity identical to those in special relativity, herein it is interpreted the changes in time and space of Lorentz transformation in special relativity with the changes in electromagnetic frequency and wavelength as in electromagnetic general relativity.

\section{Conclusions}

In this article, it reviewed the electromagnetic unification of gravitational field in the recently published book "Electromagnetic Unification of Four Forces" (ISBN978-3-659-76798-2). Based on the mutual transformation of $\gamma$ photon of high energy and the gravitational mass, the author Zi-Jian Cai and European people in television proposed the gravitational mass as the spatially localized electromagnetic structure. Later, a Zhejiang member with heavy Suzhou tone operating satellites proposed the electromagnetic orienting force from attraction longer than repulsion. Due to it as a force from the accumulative effects of both attraction and repulsion, the electromagnetic orienting force is irrelevant to the characters of charges, alike to the gravitational force. The gravitational field of Einstein's general relativity was suggested as manifesting the curve and red shift of electromagnetic wave by the electromagnetic orienting force, with changes in Einstein's physical time and space replaced by those in electromagnetic frequency and wavelength.

In addition to these main themes of electromagnetic unification of gravitational field, there are still some relevant important paradoxes and problems required to be solved. The constance for the gravitational G among various elements was interpreted as resulting from identical mass frequency for the same quark. The special relativity of Einstein has encountered new difficulties, such as the constance of electromagnetic environment adopted by the Chinese people in television to account for the constance of light speed irrelevant to the movement of illuminant in vacuum, plus the speed of neutrino of mass at least equal to that of light. Therefore, the special relativity of Lorentz transformation was explained 
by the changes in electromagnetic frequency and wavelength as in electromagnetic general relativity, with the physical time and space of Einstein of electromagnetic interactions replaced by ideal time and space without electromagnetic interactions. The US operators of satellites utilized the electromagnetic interactions of moving particles with the centric radial electrical field to demonstrate the electromagnetic interactions forming the discrepancies between the ideal time or space and electromagnetic physical time or space.

\section{Funding}

The author declares no financial support for this work.

\section{Acknowledgements}

It is herein acknowledged that Mingxun Cai paid the Open Access publication fee of this paper.

\section{Conflicts of Interest}

The authors declare no conflicts of interest regarding the publication of this paper.

\section{References}

[1] Cai, Z.-J. (2015) Electromagnetic Unification of Four Forces. Lambert Academic Publishing, Saarbrücken, Germany.

[2] Cai, Z.-J. (2016) Review of Milestone Book: Electromagnetic Unification of Four Forces. Research \& Reviews. Journal of Pure and Applied Physics, 4, 20-27.

[3] Cai, Z.-J., Beijing Member, S.C.G., European People, T.V. and Southern American People, T.V. (2015) Explaining the Mass with the Spatially Localized Electromagnetic Structure. In: Cai, Z.-J., Ed., Electromagnetic Unification of Four Forces, Lambert Academic Publishing, Saarbrücken, Germany, 34-37.

[4] Yang, C.N. and Mills, R.L. (1954) Conservation of Isotopic Spin and Isotopic Gauge Invariance. Physical Review, 96, 191-195. https://doi.org/10.1103/PhysRev.96.191

[5] Crewther, R.J. (1986) Testing the Mode of Quark Condensation. Physics Letters B, 176, 172-178. https://doi.org/10.1016/0370-2693(86)90945-7

[6] Dai, Y.B., Ding, Y.B., Huang, C.S. and Wang, C.L. (1992) Top Quark Mass in Top Quark Condensation Model. Physics Letters B, 285, 245-250. https://doi.org/10.1016/0370-2693(92)91460-Q

[7] Zhejiang Members, S.C.G. and Shanghai Member, S.C.G. (2015) The Electromagnetic Orienting Force. In: Cai, Z.-J., Ed., Electromagnetic Unification of Four Forces, Lambert Academic Publishing, Saarbrücken, Germany, 38-42.

[8] Shanghai Member, S.C.G., US People, T.V., Nanjing People, T.V., US Members, S.C.G., Southern American People, T.V., Zhejiang Members, S.C.G. and Beijing People, T.V. (2015) Explaining the Gravitational Field of General Relativity with the Electromagnetic Wave in Curve and Red Shift. In: Cai, Z.-J., Ed., Electromagnetic Unification of Four Forces, Lambert Academic Publishing, Saarbrücken, Germany, 67-72.

[9] Cai, Z.-J. (2018). San Cai Historical Memoirs in Television. Lambert Academic Pub- 
lishing, Beau Bassin, Mauritius.

[10] Yangzhou People, T.V. (2015) Explaining the Phenomenon of Light Speed in Irrelevancy to Movement in Vacuum. In: Cai, Z.-J., Ed., Electromagnetic Unification of Four Forces, Lambert Academic Publishing, Saarbrücken, Germany, 22-24.

[11] Antonello, M., Aprili, P., Baiboussinov, B., Baldo Ceolin, M., Benetti, P., Calligarich, E., Canci, N., et al. (2012) Measurement of the Neutrino Velocity with the ICARUS Detector at the CNGS Beam. Physics Letters B, 713, 17-22. https://doi.org/10.1016/j.physletb.2012.05.033

[12] Cai, Z.-J., Sports-People 2000, O.O.C., Sports-People 2004, O.O.C., Beijing Member, S.C.G., Jiang. K.T.V. and Hongkong People, T.V. (2015) Disproving the Relativity of Ideal Time and Space. In: Cai, Z.-J., Ed., Electromagnetic Unification of Four Forces, Lambert Academic Publishing, Saarbrücken, Germany, 11-16.

[13] US Members, S.C.G., British People, T.V. and Brazilian People, T.V. (2015) Explaining the Special Relativity with an Electromagnetic Method. In: Cai, Z.-J., Ed., Electromagnetic Unification of Four Forces, Lambert Academic Publishing, Saarbrücken, Germany, 29-33. 